SUBJECT AREAS:

INFORMATION TECHNOLOGY

COMPUTER SCIENCE

SCIENTIFIC REPORTS:

$3: 2993$

DOI: $10.1038 /$ srep02993

(2013)

Published:

21 October 2013

Updated:

17 November 2014

\section{ERRATUM: Identifying overlapping communities as well as hubs and outliers via nonnegative matrix factorization}

\author{
Xiaochun Cao, Xiao Wang, Di Jin, Yixin Cao \& Dongxiao He
}

This Article contains an error in the Acknowledgements section:

"This work was supported by National Basic Research Program of China (2013CB329305), National Natural Science Foundation of China (61332012, 61303110), 100 Talents Programme of The Chinese Academy of Sciences, Strategic Priority Research Program of the Chinese Academy of Sciences (XDA06030601), and Open Project Program of Key Laboratory of Symbolic Computation and Knowledge Engineering of Ministry of Education (93K172013K02)."

should read:

“This work was supported by National Natural Science Foundation of China (61332012), National Basic Research Program of China (2013CB329305), National Natural Science Foundation of China (61303110), 100 Talents Programme of The Chinese Academy of Sciences, Strategic Priority Research Program of the Chinese Academy of Sciences (XDA06030601), and Open Project Program of Key Laboratory of Symbolic Computation and Knowledge Engineering of Ministry of Education (93K172013K02)." 